\title{
Optimum Yoke Shape Designs for Enhancing Flux Density in Dual-Halbach Arrayed Permanent Magnets
}

\author{
S. Isogami, and H. Matsumoto* \\ Department of General Education, Fukushima National College of Technology, Iwaki 970-8034, Japan \\ * Department of Electric Engineering, Fukushima National College of Technology, Iwaki 970-8034, Japan
}

Dual-Halbach arrayed permanent magnets were produced with Dy-free Nd bonded permanent magnets whose external dimensions were $14 \times 14 \times 4 \mathrm{~mm}$. The dual-Halbach magnets were arranged facing different poles with a 6 -mm-distance air gap using the back yoke. The magnetic flux density at the center of the air gap was measured with a two-axis Hall prober and was also numerically calculated with the three-dimensional finite-element method. The flux density was enhanced with the front yoke by $9 \%$ compared to that without the front yoke. The front yoke also improved the irreversible flux loss after high temperature annealing. Furthermore, a $2.2 \%$ increase was successfully achieved with fragmentation of the back yoke. The calculated three-dimensional flux structure indicated that the efficient magnetic circuits with less stray flux to the outside enabled further enhancement of the flux density even in magnet arrays that involved complex multi-poles.

Key words: dual-Halbach, magnetic circuits, flux density, yoke

\section{Introduction}

Halbach arrayed permanent magnets (hereafter, called as HAMs) involve the unique features that augment the magnetic fields on one side, in contrast, cancel the magnetic field on the other side. Therefore, enhancement of the flux density on one side can be expected just as much as the flux that canceled on the other side. ${ }^{1-2)}$ In recent years, HAMs have been widely used for highly-efficient electromagnetic devices as well as mechanic devices, which are electric motors, tape recorders and undulators at synchrotron radiation facilities. ${ }^{3-8)}$

On the other hand, we have investigated smaller HAMs with several $\mathrm{mm}$ in dimension, which are equipped in present optical pickup devices for audio-visual players. The pickups need the motions to avoid sound skipping due to vibrations and/or scratches on media, and the principle to satisfy it is the electromagnetic-force generated between magnetic fields and coils. $\mathrm{Nd}-\mathrm{Fe}-\mathrm{B}$ sintered magnets with $\mathrm{Dy}$ atoms are adopted in the present pickups to provide the efficient magnetic flux. However, due to unstable supply and price rise of Dy, alternative permanent magnets are required in the future industry. We have focused on Dy-free Nd bonded-magnets (hereafter, called as BMs) as one of the proper permanent magnets, since not only the cost efficiency but also the advantages in molding small and multiplex magnets. However, the present report revealed that the BMs still have the essential issues that the flux density is much smaller than that for the $\mathrm{Nd}-\mathrm{Fe}-\mathrm{B}-\mathrm{Dy}$ sintered magnets. Therefore, we have focused on enhancing the flux density for the BMs by means of the magnet array and the optimum yokes.

Since the yokes that attached to the permanent magnets produce the magnetic circuits, one can reduce the stray flux to the outside, and enhancing the flux density can be achieved at the air gap that sandwiched by the dual magnets [see Fig. 1(a)]. The optimum yoke shapes for the standard permanent magnets, namely, the plain shaped magnets have been widely investigated so far. ${ }^{9,10)}$ On the other hand, in the case of the HAMs, investigation of the suitable yoke shapes have not been performed due to the complex multi-poles.

Therefore, the present work was intended to propose the optimum yoke shape for the dual-HAMs, and to improve the flux density at the air gap up to the value for the conventional $\mathrm{Nd}-\mathrm{Fe}-\mathrm{B}-\mathrm{Dy}$ magnets. In addition to that, analysis of the magnetic flux distribution and quantification of the irreversible flux loss after high temperature annealing were conducted to verify the effects of the proposed yoke shape.

\section{Experimental and analysis procedure}

Figure 1(a) shows the dual-HAMs produced in this study. The assembly of one HAM consists of three BMs with magnetic dipoles. Two of the BMs with dimensions of $4 \times 4 \times 14 \mathrm{~mm}$ are attached to the center BM from both sides, and the completed dimensions become $14 \times$ $14 \times 4 \mathrm{~mm}$. Here, such the size was determined based on the calculation to obtain the strongest flux focusing above the single HAM. A pair of HAMs were placed facing different poles each other with 6 -mm-distance air gap using the back yoke, resulting in the dual-HAMs. The yokes used in this study were cut from the non-oriented electrical steel sheets with $B_{\mathrm{s}}$ of $1.6 \mathrm{~T}$. The perpendicular component, namely, $z$ axis of the flux density $\left(B_{z}\right)$ and distribution of the $B_{z}$ were measured using the two-axis Hall prober at 3-mm-height with 

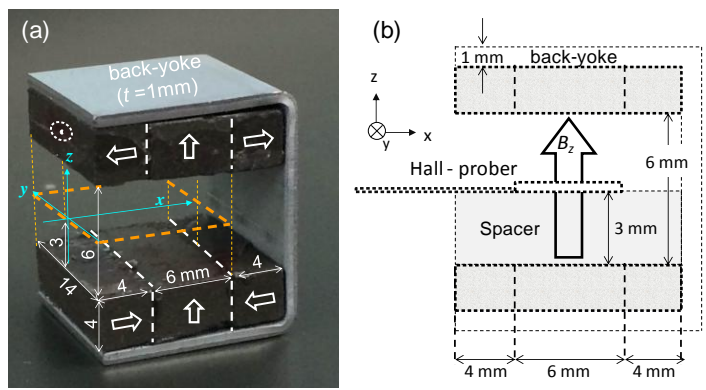

Fig. 1 (a) Photograph of dual-Halbach arrayed permanent magnets (dual-HAMs) assembly with $\mathrm{Nd}$ bonded-magnets (BMs) and attached back yoke structure. Magnetic dipoles of permanent magnets are indicated by arrows. Squares indicated by solid orange lines represent the scanning area of the two-axis Hall prober for measuring the flux density. (b) Schematic illustration showing the measurements using the two-axis Hall prober.

respect to the surface of the HAM. The dual-HAMs with the back and front yokes were annealed and cooled sequentially (see Table 1), then the irreversible flux loss was measured for the dual-HAMs without the front yoke at room temperature. While the simple estimation of the flux density at the air gap between the dual-HAMs has been proposed, ${ }^{11)}$ to analyze the detail flux distribution, we performed the numerical calculation for the static field by means of the three-dimensional finite-element method using a JMAG software package in this study.

\section{Experimental results}

\subsection{Material dependence of flux density}

Figure 2(a) shows the distribution of the $B_{\mathrm{z}}$ for the BM measured by scanning the two-axis Hall prober. The specimen in this measurement corresponds to the dual-HAM replaced by the plain shaped BMs. The distribution revealed the symmetry in $y$ axis due to the plain shaped magnet (not HAM).

Figure 2(b) shows the experimental and the calculated data for the $B_{\mathrm{z}}$ as a function of $x$ in the plane: $z=0$. We can confirm that the calculated data shows good agreement with the experimental data. In the BMs case, the parabola-like distribution appeared, and the maximum $B_{\mathrm{z}}$ of $0.29 \mathrm{~T}$ was observed at the center of $x$. The back yoke was located at $x=14$, so that the $B_{\mathrm{z}}$ for $x$ $=0$ was different from that for $x=14$. On the other hand, in the $\mathrm{Nd}-\mathrm{Fe}-\mathrm{B}-\mathrm{Dy}$ magnet case, similar distribution was observed, however, the maximum $B_{\mathrm{z}}$ reached $0.45 \mathrm{~T}$. The value was much larger than that of $\mathrm{BM}$; therefore, BMs cannot be the alternative materials of the conventional Nd-Fe-B-Dy as long as the plain in shape. (a)

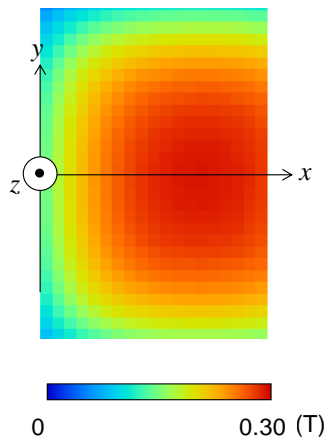

(b)

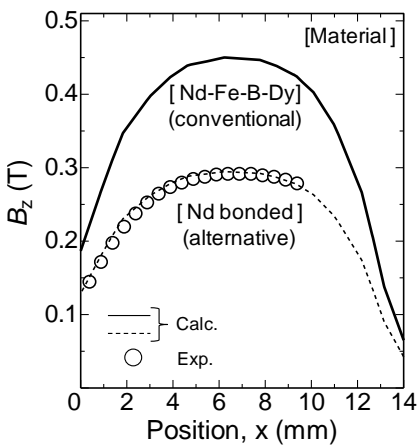

Fig. 2 (a) Distribution of flux density on $z$ axis $\left(B_{\mathrm{z}}\right)$ measured with Hall prober for plain-shaped $\mathrm{Nd}$ bonded-magnets (BMs). (b) Dependence of $B_{\mathrm{z}}$ on position of Hall prober $(x)$ on $z=0$ plane. Open circles, solid line, and dotted line correspond to the experimental data, the calculated data for $\mathrm{Nd}-\mathrm{Fe}-\mathrm{B}-\mathrm{Dy}$ conventional magnets, and for the BM. (a)

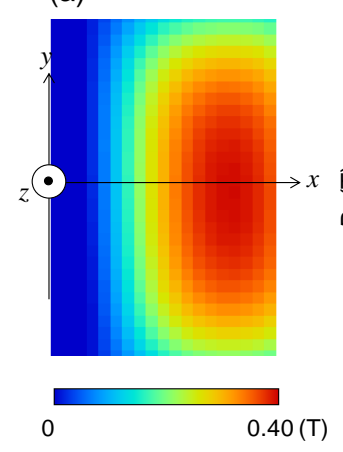

(b)

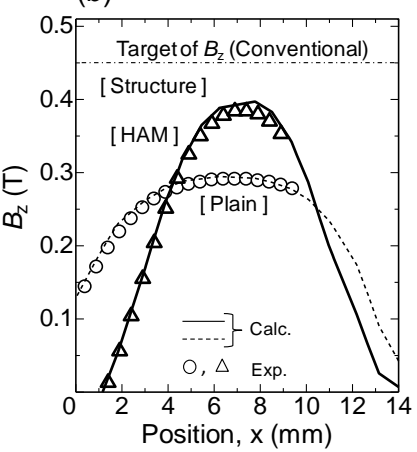

Fig. 3 (a) Distribution of flux density on $z$ axis $\left(B_{\mathrm{z}}\right)$ measured with Hall prober for dual-Halbach arrayed permanent magnets (dual-HAMs) with $\mathrm{Nd}$ bonded-magnets (BMs). (b) Dependence of $B_{\mathrm{z}}$ on the position of the Hall prober $(x)$ on $z=0$ plane. Open circles (triangles) and dotted (solid) line correspond to the experimental and calculated data for plain shaped magnets (HAMs). The chain line on the same panel represents the maximum $B_{\mathrm{z}}$ value for $\mathrm{Nd}-\mathrm{Fe}-\mathrm{B}-\mathrm{Dy}$ conventional magnets, which were the target of the present study.

\subsection{Improvement of $B_{\mathrm{z}}$ by magnet assembly}

Figure $3(\mathrm{a})$ shows the distribution of the $B_{\mathrm{z}}$ for the dual-HAM using the BMs. Comparing to the distribution for the plain shaped shown in Fig. 2(a), the flux was obviously focused along the $x$ direction, which was attributed to the basic property for HAMs.

Figure 3(b) shows the experimental and the calculated data for the $B_{\mathrm{z}}$ as a function of $x$ in the plane: $z=0$. We can confirm that the calculated data shows good agreement with the experimental data. Comparing 
(a)

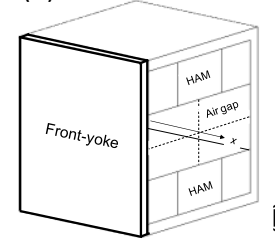

(b)

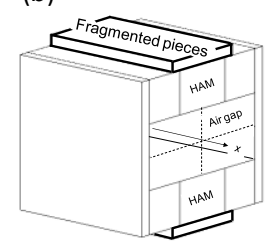

(c)

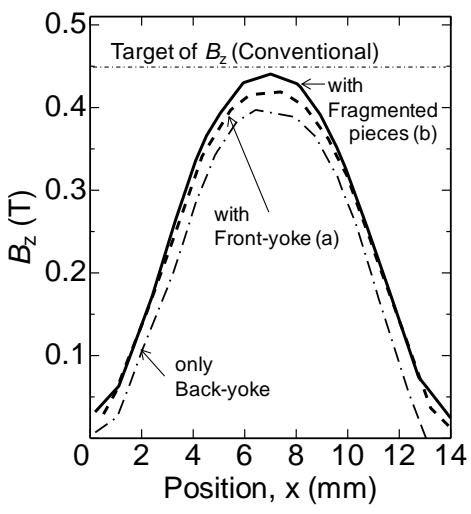

Fig. 4 Schematic of dual-HAMs (a) with front yoke and (b) with fragmented pieces. (c) Dependence of $B_{\mathrm{z}}$ on $x$. The solid, dashed, and chain lines correspond to distribution of $B_{\mathrm{z}}$ for dual-HAMs with fragmented pieces [corresponding to Fig. 4(b)], with the front yoke [corresponding to Fig. 4(a)], and with only the back yoke [corresponding to the data indicated by the solid line in Fig. 3(b)].

to the distribution for the plain shaped magnet, the dependency of $x$ was strong for the HAMs. Note that the maximum $B_{\mathrm{z}}$ for the HAM was measured to be $0.39 \mathrm{~T}$, which was $34 \%$ larger than that for the plain shaped magnet. However, the $B_{\mathrm{Z}}$ was not still enough comparing to that for the conventional Nd-Fe-B-Dy magnet, which is the attainment target in this study.

\subsection{Optimum yoke shape designs for dual-HAMs}

Figure 4(a) and 4(b) show the schematic illustrations of the dual-HAMs with the front yoke and the fragmented pieces, respectively. The fragmented pieces do not cover the whole plane of the HAMs, of which external dimensions were $14 \times 6 \times 1 \mathrm{~mm}$. Here, size of the pieces was optimized to provide the largest $B_{z}$.

Figure 4(c) shows the distribution of the $B_{\mathrm{z}}$ as a function of $x$. The similar parabola-like behaviors were observed for all the specimens, however, the maximum $B_{\mathrm{z}}$ with the front yoke was improved by $9 \%$ compared with that without the front yoke. Furthermore, almost the same maximum $B_{\mathrm{z}}$ was successfully achieved by adding the fragmented pieces, comparing to the target value. The result implied that the $\mathrm{Nd}-\mathrm{Fe}-\mathrm{B}-\mathrm{Dy}$ conventional magnets could be replaced by the BMs for the case in which the BMs were arrayed in Halbach-type and were surrounded by the fragmented yoke pieces, in spite of their less elementary $B_{z}$ comparing to the conventional $\mathrm{Nd}-\mathrm{Fe}-\mathrm{B}-\mathrm{Dy}$ magnets.

\subsection{Irreversible flux loss after high temperature annealing for dual-HAMs}

Table 1 Irreversible flux loss after high temperature annealing

\begin{tabular}{cccccc}
\hline & & \multicolumn{2}{c}{$18 \mathrm{MF}$} & \multicolumn{2}{c}{$17 \mathrm{ME}$} \\
\cline { 3 - 6 } $\begin{array}{c}\text { Temperature } \\
\text { Time }\end{array}$ & $\begin{array}{c}\text { Gap } \\
(\mathrm{mm})\end{array}$ & $\begin{array}{c}\text { Plain } \\
(\%)\end{array}$ & $\begin{array}{c}\text { HAM } \\
(\%)\end{array}$ & $\begin{array}{c}\text { Plain } \\
(\%)\end{array}$ & $\begin{array}{c}\text { HAM } \\
(\%)\end{array}$ \\
\hline $105^{\circ} \mathrm{C}, 2 \mathrm{~h}$ & 8 & -7.0 & -6.5 & & \\
\hline $100^{\circ} \mathrm{C}, 2 \mathrm{~h}$ & 6 & -4.2 & -3.3 & -1.9 & -2.2 \\
\hline $90^{\circ} \mathrm{C}, 12 \mathrm{~h}$ & 6 & -2.9 & -2.4 & & \\
\hline
\end{tabular}

Table 1 shows the comparison of irreversible flux loss for the plain shaped magnets and the dual-HAMs. The flux loss was estimated by using the simple formula: [ $B_{\mathrm{z}}$ (before annealing) $-B_{\mathrm{z}}$ (after annealing)] $/ B_{\mathrm{z}}$ (before annealing), with the measured $B_{\mathrm{z}}$ at the center of the gap. The magnet structure in this experiment corresponds to Fig. 4(a). The experiment was conducted under the several thermal conditions, gap length and two kinds of materials such as $18 \mathrm{MF}$ (BM investigated in this study) and 17ME (BM with larger coercive force than $18 \mathrm{MF}$ ). For all the annealing conditions as well as the gap length, the flux loss in the HAMs was more improved comparing to the plain shaped magnets. In the material with high coercivity case, $17 \mathrm{ME}$, much less value of $-1.9 \%(-2.2 \%)$ was observed for the plain shaped magnets (HAMs). The front yoke could achieve the efficient magnetic circuit, resulting in high permeance even in the HAMs. From that reason, the flux loss in HAMs might be suppressed. (a)

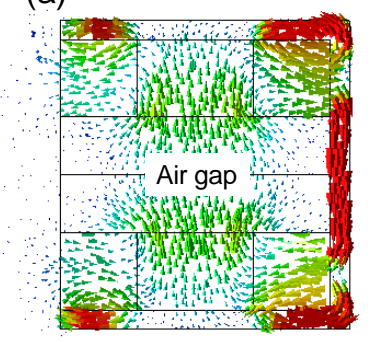

(c)

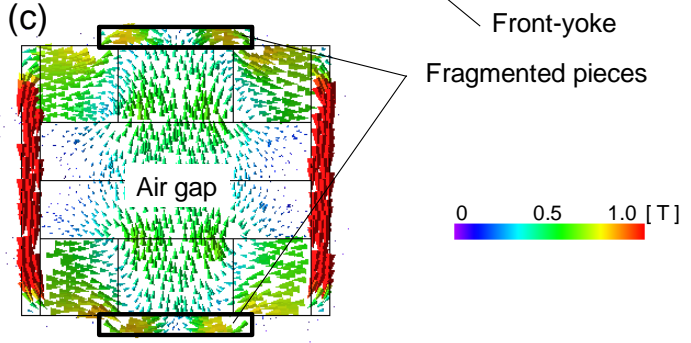

(b)

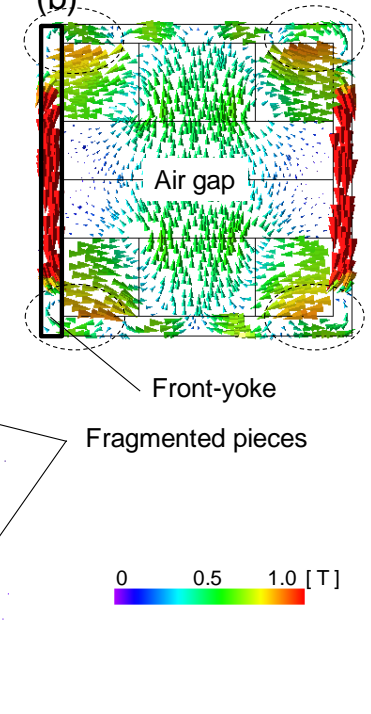

Fig. 5 Vectors of flux density calculated with the three-dimensional finite-element method using JMAG software for dual-HAMs with (a) only back yoke, (b) front yoke, and (c) fragmented pieces. The four circles with dotted lines indicate where closed eddy flux appeared with the flux direction opposite to periphery. 


\section{Discussion}

We found that not only the front yoke but also the fragmented pieces helped the dual-HAMs enhance the flux density in this study. To discuss the mechanism for that, the three-dimensional flux vectors were described by means of three-dimensional finite-element method. Figure 5(a) shows the vector mapping with only the back yoke. The stray flux out of the dual-HAM was seen to be tangible at the opposite side of the back yoke. On the other hand, the flux flows into the front yoke, and returned to the other side of the magnet in the case that the front yoke was attached [see Fig. 5(b)]. However, the closed eddy flux was appeared at the four corners of the dual-HAM, of which direction was opposite to the peripheral flux. The fragmented pieces can prevent the generation of the eddy flux, since the magnetic circuit of the yokes turns to be discontinuous [see Fig. 5(c)]. Judging from the slight stray flux that appeared near the fragmented pieces, the flux density in the yokes might exceed their magnetic saturation. Therefore, not all the component of flux loss can return back to the other side of HAM. Nevertheless, further improvement was successfully achieved by addition of the fragmented pieces. Such the facts provided the authors the conclusions that the fragmented pieces were optimum yoke shape for the dual-HAMs.

\section{Summary}

Studies on the optimum yoke shape for enhancing the flux density in the dual-HAMs were performed by means of the experiments with two-axis Hall prober as well as the numerical calculation with the three-dimensional finite-element method. To achieve the maximum flux density of the conventional $\mathrm{Nd}-\mathrm{Fe}-\mathrm{B}-\mathrm{Dy}$ magnets, adding the front yoke and the fragmented pieces were indispensable. The calculation for the flux vectors revealed that the stray flux returns along the front yoke. Moreover, preventing the generation of the eddy flux at the four corners of the dual-HAMs were the key factors to achieve more improvement. The irreversible demagnetization after high temperature annealing was measured for dual-HAMs with the front-yoke and the back yoke. We confirmed that the demagnetization was suppressed in the dual-HAMs rather than the plain shaped magnet, which might imply increase in the permeance factor of the dual-HAMs due to the front-yokes and the back yokes.

\section{References}

1) K. Halbach: Nucl. Instrum. and Methods., 169, 1 (1980).

2) K. Halbach: Nucl. Instrum. and Methods., 187, 109 (1981).

3) J.C. Mallinson: IEEE Trans. Magn., 9, 678 (1973).

4) F. Bloch, O. Cugat, G. Meunier and J.C. Toussaint: IEEE Trans. Magn., 34, 2465 (1998).

5) M. Kumada, E.I. Autokhin, Y. Iwashita, M. Aoki and E. Sugiyama: IEEE Trans. Appl. Supercond., 14, 1287 (2004).

6) C.R. Saha, Paul H. Riley, J. Paul, Z. Yu, A.J. Jaworski and C.M. Johnson: Sens. Actuators, A, 178, 179 (2012).

7) M. Nakamura, M. Koyama and M. Kobayashi: The $3^{r d}$ Magneto Electron. Int. Symp, 2, 48 (2004).

8) A. Sarwar, A. Nemirovski and B. Shapiro: J. Magn. Magn. Mater., 324, 742 (2012).

9) O. Okuno, S. Ishikawa, F.T. Iimuro, Y. Kinouchi, H. Yamada, T. Nakano, H. Hamanaka, N. Ishihata, H. Mizutani and M. Ai: Dent. Mat. J., 10, 172 (1991).

10) J. Iniguez and V. Raposo: J. Magn. Magn. Mater., 322, 1673 (2010).

11) M. Morishita, S. Yokoyama and R. Okuyama: IEEJ Trans. Ind. Appl., 132, 596 (2012).

Received Oct. 2, 2014; Accepted Nov. 14, 2014 